\title{
ARTICLE
}

Received 11 Dec 2014 | Accepted 27 Apr 2015 | Published 19 Jun 2015

\section{Atomically thin resonant tunnel diodes built from synthetic van der Waals heterostructures}

Yu-Chuan Lin', Ram Krishna Ghosh², Rafik Addou ${ }^{3}$, Ning Lu ${ }^{3}$, Sarah M. Eichfeld ${ }^{1}$, Hui Zhu ${ }^{3}$, Ming-Yang Li ${ }^{4}$, Xin Peng ${ }^{3}$, Moon J. Kim ${ }^{3}$, Lain-Jong Li ${ }^{5}$, Robert M. Wallace ${ }^{3}$, Suman Datta ${ }^{2}$ \& Joshua A. Robinson ${ }^{1}$

Vertical integration of two-dimensional van der Waals materials is predicted to lead to novel electronic and optical properties not found in the constituent layers. Here, we present the direct synthesis of two unique, atomically thin, multi-junction heterostructures by combining graphene with the monolayer transition-metal dichalcogenides: molybdenum disulfide $\left(\mathrm{MoS}_{2}\right)$, molybdenum diselenide $\left(\mathrm{MoSe}_{2}\right)$ and tungsten diselenide $\left(\mathrm{WSe}_{2}\right)$. The realization of $\mathrm{MoS}_{2}-\mathrm{WSe}_{2}$-graphene and $\mathrm{WSe}_{2}-\mathrm{MoS}_{2}$-graphene heterostructures leads to resonant tunnelling in an atomically thin stack with spectrally narrow, room temperature negative differential resistance characteristics.

\footnotetext{
${ }^{1}$ Department of Materials Science and Engineering and Center for 2-Dimensional and Layered Materials, The Pennsylvania State University, University Park, Pennsylvania 16802, USA. ${ }^{2}$ Department of Electrical Engineering, The Pennsylvania State University, University Park, Pennsylvania 16802, USA. ${ }^{3}$ Department of Materials Science and Engineering, The University of Texas at Dallas, Richardson, Texas 75080, USA. ${ }^{4}$ Institute of Atomic and Molecular Sciences, Academia Sinica, Taipei 10617, Taiwan. ${ }^{5}$ Physical Science and Engineering, King Abdullah University of Science and Technology, Thuwal 23955-6900, Saudi Arabia. Correspondence and requests for materials should be addressed to J.A.R. (email: jrobinson@psu.edu).
} 
$\mathrm{R}$ esonant tunnelling of charge carriers between two spatially separated quantum states can lead to a unique current transport phenomenon known as negative differential resistance $(\mathrm{NDR})^{1,2}$. This is a key feature for novel nanoelectronic circuits that utilize bistability and positive feedback, such as novel memories, multi-valued logic, inductorfree compact oscillators and many other not-yet-realized electronic applications ${ }^{3,4}$. However, realizing spectrally narrow NDR in a resonant tunnelling diode (RTD) at room temperature has been challenging due to carrier scattering related to interfacial imperfections, which are unavoidable in traditional semiconductor heterostructures synthesized using advanced epitaxial growth techniques ${ }^{5}$. Two-dimensional (2D) materials ${ }^{6,7}$, with no out-of-plane chemical bonding and pristine interfaces, presents an appealing alternative to traditional semiconductors, and could ultimately eliminate the interfacial imperfections that limit room temperature NDR performance to date. Since 2004 (ref. 6), the overwhelming majority of electronic transport and stacked in 2D materials has been reported using mechanically exfoliated flakes ${ }^{8}$. Recently, there has been a concerted effort to directly synthesize layered transition-metal dichalcogenides (TMDs), with powder vapourization $^{9-11}$ synthesis paving the way for direct growth of atomically thin structures ${ }^{9-14}$. Beyond monolayer (ML) TMDs, van der Waals (vdW) heterostructures (heterogeneous stacks of dissimilar atomic layers) have been predicted to lead to novel electronic properties not found in their constituent layers ${ }^{15}$, where their realization has primarily come from mechanical exfoliation and stacking ${ }^{16-19}$. Manual stacking has provided experimental verification of electronic bandgap modulations and strong interlayer coupling 20 , but it can also lead to interface contamination ${ }^{19}$ that introduces additional scattering mechanisms and inhibits the NDR. Therefore, a synthetic route to achieve vdW heterostructures with pristine interfaces will be a critical step in the advancement of the field.

Here we present the direct synthesis of $\mathrm{MoS}_{2}-\mathrm{WSe}_{2}$-graphene and $\mathrm{WSe}_{2}-\mathrm{MoSe}_{2}$-graphene heterostructures employing a combination of oxide powder vapourization and metal-organic chemical vapour deposition (MOCVD). We not only demonstrate that these heterostructures exhibit the same interlayer electronic coupling found in mechanically exfoliated heterostructures ${ }^{20-22}$, but also show that they exhibit unique electronic transport properties not typically found in exfoliated structures. We discover that direct grown heterostructures exhibit resonant tunnelling of charge carriers, which leads to sharp NDR at room temperature. Importantly, we identify that the peak of the resonant tunnelling can be tuned by modifying the stacking order or layer composition, which will be a powerful tool towards engineering heterostructures for ultra-low power electronic devices.

\section{Results}

Formation of vertical vdW heterostructures. The heterostructure is achieved by sequentially growing two dissimilar TMD MLs on multilayer (three layers) epitaxial graphene (EG) (Fig. 1a $)^{23}$. The individual TMD layers are grown ex situ via powder vapourization or MOCVD. Tungsten diselenide is synthesized using both routes: tungsten trioxide $\left(\mathrm{WO}_{3}\right)$ and selenium (Se) powders for the powder vapourization route ${ }^{24}$, and tungsten hexacarbonyl $\left(\mathrm{W}(\mathrm{CO})_{6}\right)$ and dimethylselenium $\left(\left(\mathrm{CH}_{4}\right)_{2} \mathrm{Se}\right)$ for the MOCVD route ${ }^{25}$. Molybdenum disulfide is grown via vapourization of molybdenum trioxide $\left(\mathrm{MoO}_{3}\right)$ and sulfur ${ }^{10}$. The heterostructure synthesis process is summarized in Fig. 1. The first TMD layer of the heterostructure, $\mathrm{WSe}_{2}$ or $\mathrm{MoS}_{2}$, is grown on tri-layer EG (Fig. 1a) at $950{ }^{\circ} \mathrm{C}$ and $750{ }^{\circ} \mathrm{C}$ for $\mathrm{WSe}_{2}-\mathrm{EG}$ (Fig. 1b) and $\mathrm{MoS}_{2}-\mathrm{EG}$ (Fig. 1c,d), respectively. Following this first TMD growth step, the surface coverage of the $\mathrm{WSe}_{2}$ or $\mathrm{MoS}_{2}$ on EG is typically $>60 \%$, with a lateral size of $2 \mu \mathrm{m}$ and $300 \mathrm{~nm}$ for $\mathrm{WSe}_{2}$ and $\mathrm{MoS}_{2}$, respectively. Subsequently, the $\mathrm{MoS}_{2}-\mathrm{WSe} \mathrm{H}_{2}-\mathrm{EG}$ vertical heterostructure is created via a second ex situ growth of $\mathrm{MoS}_{2}$ on $\mathrm{WSe} e_{2}-\mathrm{EG}$ at $750^{\circ} \mathrm{C}$ (Fig. 1c). Similar to our previous work ${ }^{26}$, we find that wrinkles in the graphene as well as defects and edges within the $\mathrm{WSe}_{2}$ promote vertical growth of the $\mathrm{MoS}_{2}$, and $\mathrm{ML} \mathrm{MoS}_{2} / \mathrm{WSe}_{2}$ is primarily achieved in pristine regions of $\mathrm{WSe}_{2}$ (Fig. 1c and Supplementary

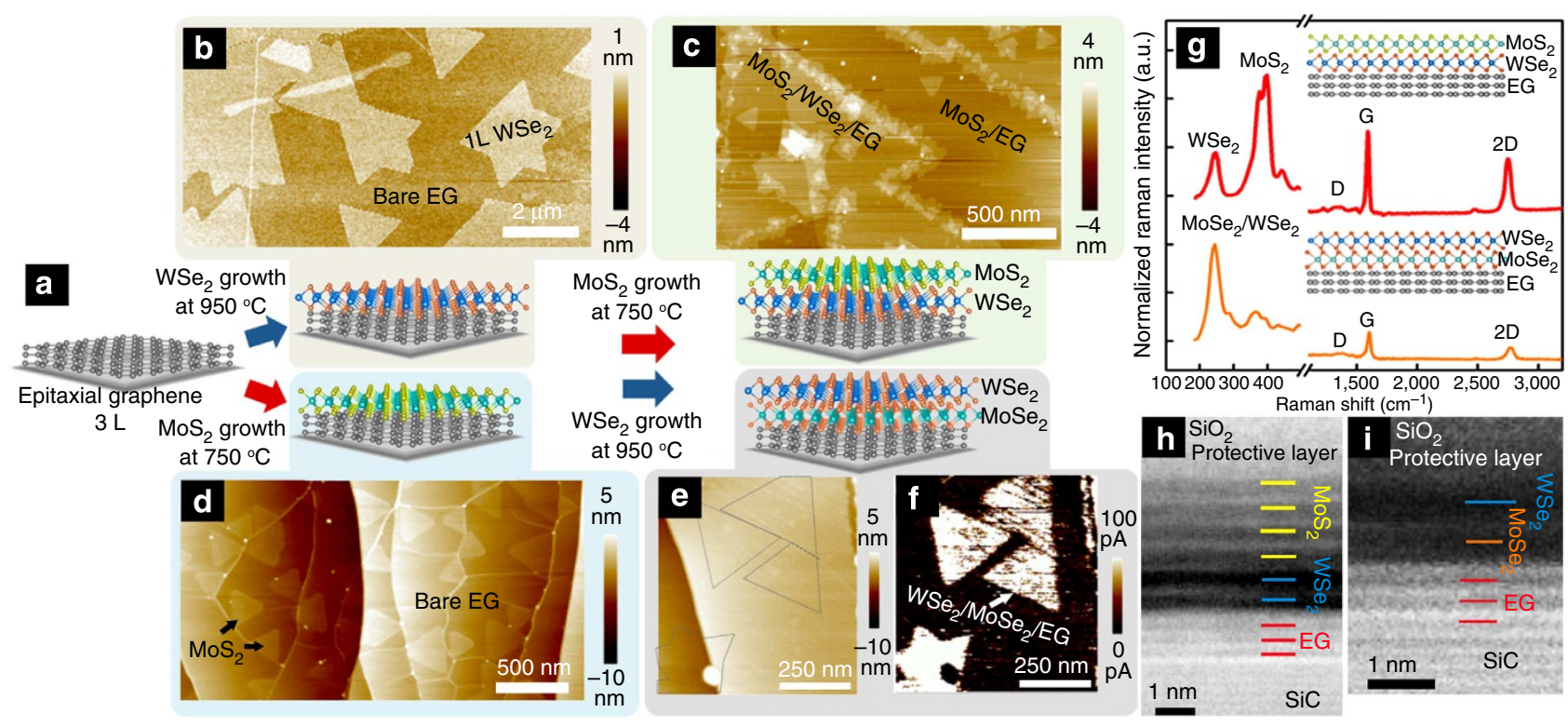

Figure 1 | The formation of vdW heterostructures. $\mathrm{MoS}_{2}-\mathrm{WSe} \mathrm{e}_{2}-\mathrm{EG}$ vertical heterostructures begins with the synthesis of (a) $3 \mathrm{~L} E \mathrm{EG}$ from SiC followed by (b) vapour transport or MOCVD of $\mathrm{WSe}_{2}$ and (c) vapour transport of $\mathrm{MoS}_{2}$. WSe $\mathrm{W}_{2}-\mathrm{MoSe}_{2}-\mathrm{EG}_{\text {heterostructures are similarly grown, except when (d) MoS }}$ is grown first on $\mathrm{EG}$ followed by (e) growth of the $\mathrm{WSe}_{2}$, a Se-S ion exchange occurs, leading to the formation of $\mathrm{MoSe}_{2}$ from the original MoS 2 layer. The $\mathrm{MoSe}_{2}$ domains are difficult to topographically identify; however, (f) conductive AFM clearly delineates their location due to enhanced tunnelling at the heterostructures. Raman $(\mathbf{g})$ indicates that preservation of the graphene has occurred during the synthesis process, and Scanning TEM (h,i) confirms that the stacked structures exhibit pristine interfaces, with no intermixing of Mo-W or S-Se after synthesis. 

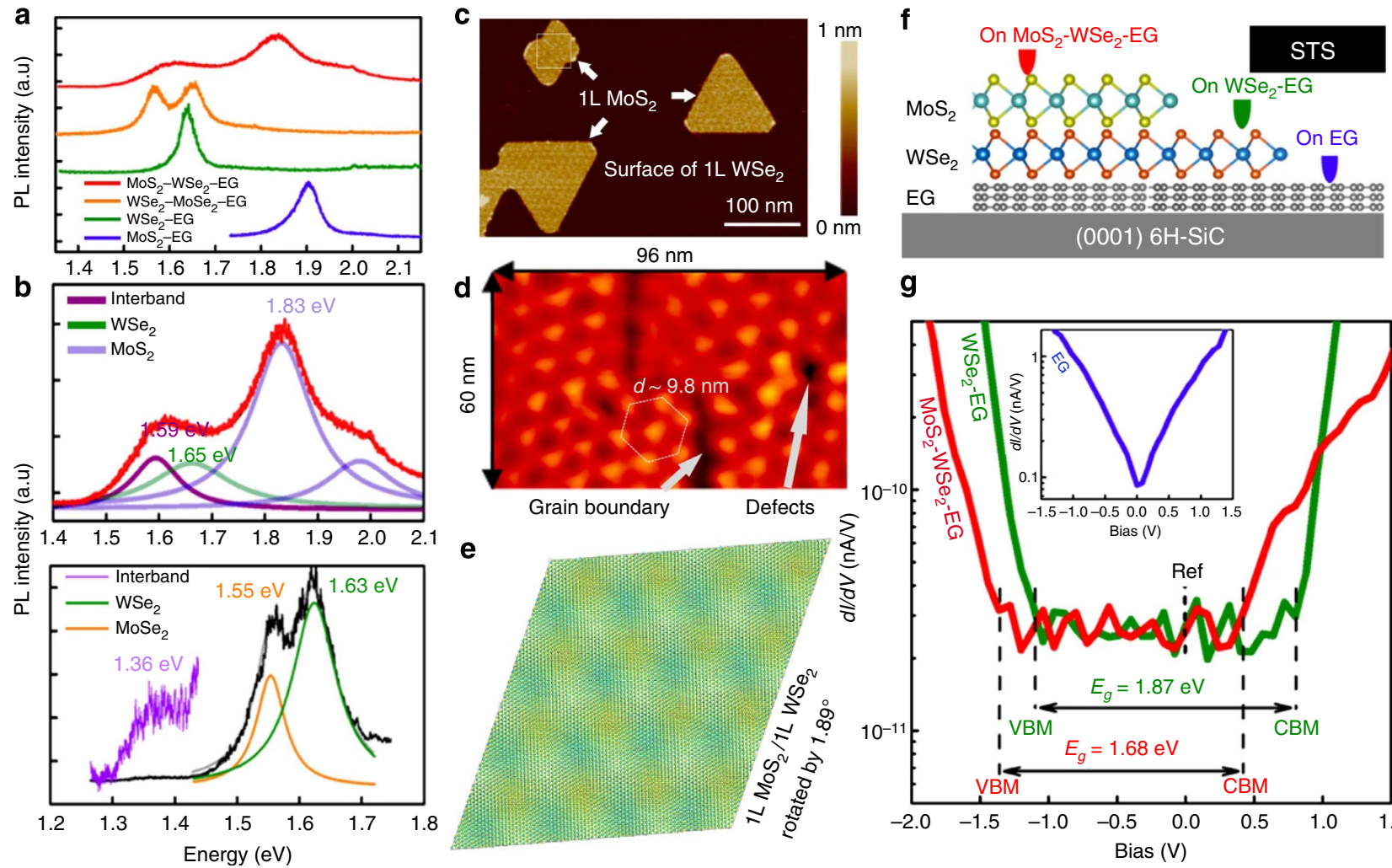

g

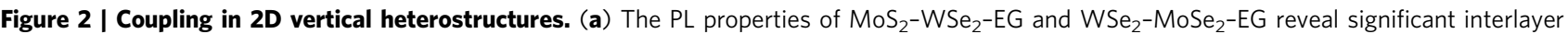
coupling, where the (b) $\mathrm{MoS}_{2}-\mathrm{WSe} e_{2}-E G$ and WSe $-\mathrm{MoSe}_{2}-E G$ exhibit the intrinsic PL peaks corresponding to $\mathrm{MoS}_{2}, \mathrm{MoSe}_{2}$ and WSe $\mathrm{W}_{2}$, and also exhibit interband PL peaks at 1.59 and $1.36 \mathrm{eV}$, where the excitation wavelength $(\lambda)$ is $488 \mathrm{~nm}$ and $633 \mathrm{~nm}$ for $\mathrm{MoS}_{2}-\mathrm{WSe}_{2}-\mathrm{EG}$ and $\mathrm{WSe} \mathrm{e}_{2}-\mathrm{MoSe}_{2}$-EG, respectively. (c) The moiré patterns acquired via AFM in $\mathrm{MoS}_{2}$ on $\mathrm{WSe}_{2}$ indicates an alignment of nearly either $0^{\circ}$ or $180^{\circ}$ between the top and bottom layer, and (d) STM confirms the moire pattern with a lattice constant equal to $(9.8 \pm 0.4) \mathrm{nm}$. This structure can be reproduced theoretically (e) when the misorientation angle between these layers is $\sim 1.9^{\circ}$. The continuity of the Moiré pattern is interrupted by the formation of a grain boundary and point defects, as indicated in the STM image. (f,g) STS on $\mathrm{MoS}_{2}-\mathrm{WS} \mathrm{e}_{2}-\mathrm{EG}, \mathrm{WSe} \mathrm{e}_{2}-\mathrm{EG}$ and $\mathrm{EG}$ (g, inset) provide evidence that the bandgap of the double junction heterostructure $\left(\mathrm{MoS}_{2}-\mathrm{WS} e_{2}-\mathrm{EG}\right)$ is smaller than that of the single-junction ( $\mathrm{WS} \mathrm{e}_{2}-\mathrm{EG}$ ) heterostructure. The positions of conduction band minimum $(C B M)$, valence band maximum (VBM), and quasi-particle bandgap $E_{g}$ of $\mathrm{WSe}_{2}$ on $E G$ and bilayer on $E G$ are marked.

Fig. 1a) ${ }^{26}$. The formation of the $\mathrm{WSe}_{2}-\mathrm{MoSe}_{2}-\mathrm{EG}$ heterojunction occurs during growth of $\mathrm{WSe}_{2}$ on $\mathrm{MoS}_{2}$. During the synthesis, a selenium-sulfur ion exchange occurs when the $\mathrm{MoS}_{2}$ is exposed to the selenium vapour just prior to the growth of $\mathrm{WSe}_{2}$ at $950^{\circ} \mathrm{C}$ for $45 \mathrm{~min}^{27}$. Standard topographic characterization via atomic force microscopy (AFM) cannot clearly identify the location of the heterostructures (Fig. 1f); however, conductive AFM (CAFM) with platinum $(\mathrm{Pt})$ tip $^{28}$ provides a means to map the $\mathrm{WSe}_{2}-\mathrm{MoSe}_{2}-\mathrm{EG}$ junctions and adjacent $\mathrm{WSe} \mathrm{e}_{2}-\mathrm{EG}$ regions due to a difference in heterostructure conductivity (Fig. If and Supplementary Fig. 1c,d).

Raman spectroscopy and transmission electron microscopy (TEM) confirm the formation of crystalline, vertical heterostructures (Fig. 1g-i and Supplementary Figs 1-3). A large fraction of the EG remains nearly defect free following the sequence of TMD growths; however, there are regions of increased defectiveness due to either partial passivation of the graphene/SiC buffer layer ${ }^{23}$ or formation of thick TMD layers ${ }^{26}$. Raman spectroscopy (see Supplementary Fig. 3) also confirms presence of significant fractions of $\mathrm{ML} \mathrm{WSe} \mathrm{W}_{2}\left(E_{2 \mathrm{~g}} / A_{1 \mathrm{~g}}\right.$ at $250 \mathrm{~cm}^{-1}$ and 2LA at $\left.263 \mathrm{~cm}^{-1}\right)^{24}$ and $\operatorname{MoS}_{2}\left(E_{2 \mathrm{~g}}\right.$ at $383 \mathrm{~cm}^{-1}$ and $A_{1 \mathrm{~g}}$ at $\left.404 \mathrm{~cm}^{-1}\right)^{26}$, as well as ML $\mathrm{MoSe}_{2}$ $\left(A_{1 \mathrm{~g}} \text { at } 240 \mathrm{~cm}^{-1} \text { and } E^{1}{ }_{2 \mathrm{~g}} \text { at } 284 \mathrm{~cm}^{-1}\right)^{27}$. X-ray photoelectron spectroscopy (see Supplementary Fig. 4 and Supplementary Table 1) also corroborates the absence of any interaction between the two TMDs or graphene, and indicates that the $\mathrm{MoS}_{2}$ exhibits an n-type behaviour, while the $\mathrm{WSe}_{2}$ layer shows a p-type behaviour. Scanning TEM (Fig. 1h,i) also verifies the heterostructure is not a manifestation of the alloying of the constituent TMDs, but indeed are unique layers with pristine interfaces with atomic precision. In the case of $\mathrm{MoS}_{2}-\mathrm{WSe} \mathrm{e}_{2}-\mathrm{EG}$, we have focused on a multilayer region of $\mathrm{MoS}_{2}-\mathrm{WSe}_{2}$ to ensure pristine layer formation beyond the ML structure (see Supplementary Fig. 2); however, all electrical characterization presented later is on ML heterostructures. The clean interface between MLs can be observed easily using high resolution scanning TEM. The $\mathrm{WSe}_{2}-\mathrm{MoSe}_{2}-\mathrm{EG}$ ordering is confirmed by comparing the intensity with that of bilayer $\mathrm{WSe} \mathrm{e}_{2}-\mathrm{EG}$ due to the similar atomic number between $\mathrm{W}$ and $\mathrm{Mo}$ atom (see Supplementary Fig. 2). Unlike vertical heterostructures based on a single chalcogen (that is, $\left.\mathrm{MoS}_{2} / \mathrm{WS}_{2}\right)^{29}$, the ordered layering does not occur when we attempt to grow a vertical structure based on heterogeneous layers where $M_{1} \neq M_{2}$ and $X_{1} \neq X_{2}$ $(M=\mathrm{Mo}, \mathrm{W} ; X=\mathrm{S}, \mathrm{Se})$ on ' $3 \mathrm{D}$ ' substrates such as sapphire or $\mathrm{SiO}_{2}$ (see Supplementary Fig. 5). Instead, all attempts to grow such a structure results in alloying or lateral heterostructures of the layers. Therefore, we hypothesize that EG plays a critical role in the formation of atomically precise vdW heterostructures, where $M_{1} \neq M_{2}$ and $X_{1} \neq X_{2}$ by providing an atomically smooth surface that is free of dangling bonds, enabling mobility on the surface for TMD layer growth. Sapphire and $\mathrm{SiO}_{2}$ surfaces exhibit high surface roughness, dangling bonds, and are therefore more likely to impede surface diffusion, which catalyzes the alloying process. 


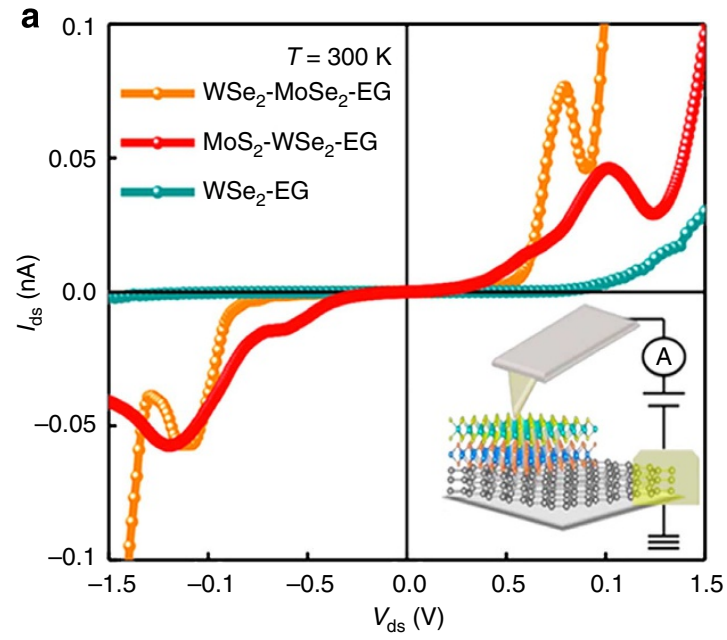

b

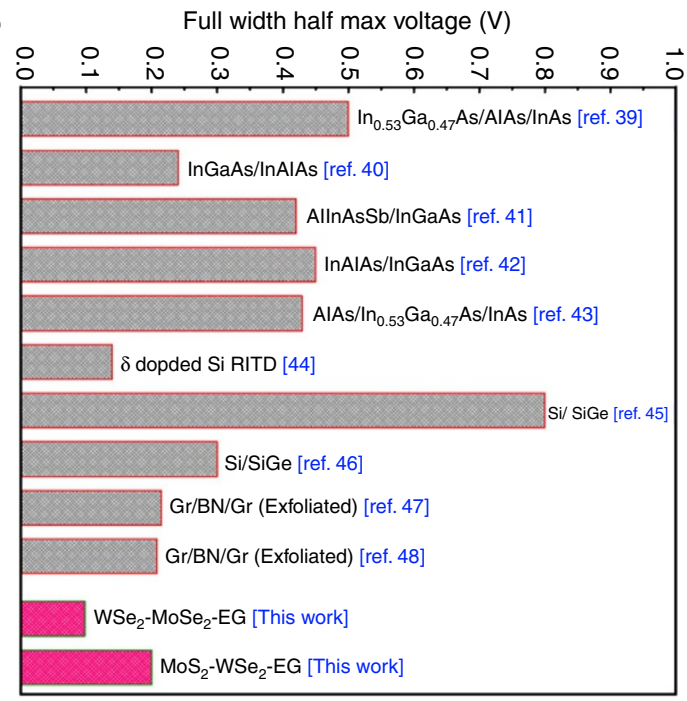

Figure 3 | Resonant tunnelling and negative differential resistance in atomically thin layers. (a) Experimental $I-V$ traces for different combination of dichalcogenide-graphene interfaces demonstrating NDR. The inset shows schematic of the experimental set-up for the I-V measurement in this layered system. (b) Comparison of full width at half maximum voltage of the NDR from this work with other reported results in room temperature ${ }^{40-49}$

Interlayer coupling. ML-semiconducting TMDs exhibit a direct optical bandgap $\left(E_{\mathrm{opt}}\right)\left(\mathrm{MoS}_{2}\right.$ at $1.8 \sim 1.9 \mathrm{eV}, \mathrm{MoSe}_{2}$ at $1.55 \mathrm{eV}$, and $\mathrm{WSe}_{2}$ at $\left.1.6 \sim 1.65 \mathrm{eV}\right)^{30}$; therefore, photoluminescence (PL) spectroscopy (Fig. 2a,b) can provide evidence of electronic coupling between the layers. In addition to the typical PL peaks from the direct bandgap transition within the individual layers, the PL spectra of the heterostructures exhibit the presence of interlayer excitons at $1.59 \mathrm{eV}$ for $\mathrm{MoS}_{2}-\mathrm{WSe}_{2}-\mathrm{EG}$ and $1.36 \mathrm{eV}$ for $\mathrm{WSe}_{2}-\mathrm{MoSe}_{2}-\mathrm{EG}$ (see Fig. 2a,b). In this case, the $\mathrm{MoS}_{2}-\mathrm{WSe}_{2}$ and $\mathrm{WSe}_{2}-\mathrm{MoSe}_{2}$ junctions exhibit type II band alignment ${ }^{15,20,21,31}$, where electrons in the $\mathrm{WSe}_{2}$ conduction band transfer to the conduction band of $\mathrm{MoS}_{2}\left(\mathrm{MoSe}_{2}\right)$ and the excited holes in $\mathrm{MoS}_{2}\left(\mathrm{MoSe}_{2}\right)$ valence band transfer to the valence band of $\mathrm{WSe}_{2}$. Consistent with manually stacked heterojunctions $s^{20,21}$, the position of the PL peak is due to interlayer exciton recombination, which confirms the electronic coupling at the heterojunction between the two ML TMDs.

Additional evidence of coupling comes from the topographical information of the heterostructures. Similar to graphene-hBN heterostructures ${ }^{32}$, Moiré patterns of $\mathrm{MoS}_{2}-\mathrm{WSe}_{2}$ are observed in tapping-mode AFM, which are qualitatively consistent with rotation angles of $\sim 0$ or $180^{\circ}$ between $\mathrm{MoS}_{2}$ and $\mathrm{WSe}_{2}$. Furthermore, scanning tunnelling microscopy/spectroscopy (Fig. 2d) confirms the presence of a Moire pattern produced by the misorientation of $\mathrm{MoS}_{2}$ relative to the underlying $\mathrm{WSe}_{2}$ layer. The lattice constant of the Moire pattern is $9.8 \pm 0.4 \mathrm{~nm}$, which corresponds to a misorientation angle of $\sim 1.9^{\circ}$. Modelling the heterostructure with this misorientation produces a consistent Moiré pattern, with a slightly smaller lattice constant of $9.6 \mathrm{~nm}$ (Fig. 2e). While the mechanical stacking technique leads to a variety of rotation angles between layers ${ }^{20}$, the direct growth of $\mathrm{vdW}$ layers using our approach appears to have a strict rotational alignment, which may be critical for achieving optimal coupling between the layers ${ }^{33,34}$.

Scanning tunnelling spectroscopy further provides evidence that the quasi-particle bandgap of $\mathrm{MoS}_{2}-\mathrm{WSe}_{2}-\mathrm{EG}$ is significantly smaller than its $\mathrm{WSe}_{2}$-EG counterpart (Fig. 2f,g, and Supplementary Fig. 6). Based on STS, we infer that, for $\mathrm{WSe}_{2}-\mathrm{EG}$, the conduction band minimum is at a sample bias of $+0.71 \pm 0.08 \mathrm{~V}$ and the valence band maximum is at $-1.11 \pm 0.02 \mathrm{~V}$ (green curve in Fig. $2 \mathrm{~g}$ ). This indicates that the quasi-particle bandgap $\left(E_{\mathrm{g}}\right)$ of $\mathrm{WSe}_{2}$ is $1.83 \pm 0.05 \mathrm{eV}$, which is higher than $E_{\mathrm{opt}}(1.63 \mathrm{eV})$ due to the large excitonic binding energy in 2D TMDs ${ }^{14,22,31,35}$. On the other hand, $\mathrm{MoS}_{2}-\mathrm{WSe}_{2}-$ EG exhibits a conduction band minimum at $+0.34 \pm 0.03 \mathrm{~V}$ and valence band maximum at $-1.31 \pm 0.03 \mathrm{~V}$, indicating a quasiparticle interlayer $E_{\mathrm{g}}$ of $1.65 \mathrm{eV} \pm 0.02 \mathrm{~V}$, which is slightly larger than its interlayer $E_{\text {opt }}$ at $1.59 \mathrm{eV}$ (Fig. 2b) but smaller than the $E_{\text {opt }}$ in $1 \mathrm{~L} \mathrm{MoS}_{2}-\mathrm{EG}^{22,31}$. Mapping the tunnel current density of $\mathrm{WSe}_{2}-\mathrm{EG}$ and $\mathrm{WSe}_{2}-\mathrm{MoSe}_{2}-\mathrm{EG}$ heterostructures via $\mathrm{CAFM}^{28,36}$ (Fig. If and Supplementary Fig. 1) provides strong evidence that tunnelling is much more readily achieved in $\mathrm{WSe}_{2}-\mathrm{MoSe}_{2}-\mathrm{EG}$ at a tip bias of $1.5 \mathrm{~V}$, indicating a smaller, resonance tunnelling or both may be occurring. Finally, we note that defects, such as grain boundaries and vacancies disrupt the continuity of the Moiré pattern, further emphasizing that imperfections in layers or the interface will significantly impact the electronic behaviour of $\mathrm{vdW}$ heterostructures (Fig. 2d).

Vertical transport. Room temperature current-voltage measurements through the heterostructure (carried out via CAFM) do not exhibit the traditional $\mathrm{p}-\mathrm{n}$ junction diode-like transport found in mechanically exfoliated dichalcogenide structures or direct grown single-junction (that is, $\mathrm{WSe}_{2}-\mathrm{EG}$ ) structures ${ }^{20,26,37}$. Instead, we find that, following a 'soft' turn-on, the current exhibits a peak at a certain bias voltage $\left(V_{\text {peak }}=+1.1\right.$ and +0.7 for $\mathrm{MoS}_{2}-\mathrm{WSe}_{2}-\mathrm{EG}$ and $\mathrm{WSe}_{2}-\mathrm{MoSe}_{2}-\mathrm{EG}$, respectively), then decreases to a minimum before undergoing a 'hard' turn-on with exponential current increase (Fig. 3a). The peak-to-valley current ratio is 1.9 for $\mathrm{MoS}_{2}-\mathrm{WSe}_{2}-\mathrm{EG}$ and 2.2 for $\mathrm{WSe}_{2}-\mathrm{MoSe}_{2}-\mathrm{EG}$ (Fig. 3a and Supplementary Fig. 7, and Supplementary Table 2), which is comparable to traditional RTDs ${ }^{1-5}$.

\section{Discussion}

We have demonstrated the direct synthesis of unique multijunction heterostructures based on graphene (EG on $\mathrm{SiC}$ ), $\mathrm{MoS}_{2}$, $\mathrm{MoSe}_{2}$ and $\mathrm{WSe}_{2}$ that yields pristine interlayer gaps and leads to the first demonstration of resonant tunnelling in a atomically thin synthetic stack with the spectrally narrowest room temperature NDR characteristics. Importantly, this work indicates that NDR at room temperature only occurs in TMD heterostructures with truly pristine interfaces, which has been recently corroborated with manually stacked heterostructures where NDR is only evident at liquid nitrogen temperatures ${ }^{20,38,39}$. This is due to 
resonant tunnelling being highly sensitive to interfacial perturbations such as defects or 'residue' from the transfer process, emphasizing the importance of direct synthesis of multijunction TMD heterostructures for vertical quantum electronics applications. Interestingly, the room temperature full width at half maximum of the NDR in this work is more spectrally narrow than their '3D' semiconductor counterparts (silicon, germanium, III-V) and manually stacked graphene-boron nitride-graphene (Gr-hBN-Gr) heterostructures (Fig. 3b and Supplementary Note 7$)^{40-49}$. This suggests that the interface of the directly grown vdW heterostructures is superior to that of many previously reported RTD structures.

\section{Methods}

EG synthesized from $6 \mathrm{H}-\mathrm{SiC}$. Graphene is synthesized on $1 \mathrm{~cm}^{2}$ squares of $6 \mathrm{H}-\mathrm{SiC}(0001)$ in a graphite crucible ${ }^{23}$. The $6 \mathrm{H}-\mathrm{SiC}$ substrate was annealed in $\mathrm{H}_{2}$ at $1,500^{\circ} \mathrm{C}$ for $10 \mathrm{~min}$ to clean substrate surface prior to graphene growth. At this stage the chamber pressure is 700 torr under a $\mathrm{H}_{2}$ ( 50 s.c.c.m)/ $\mathrm{Ar}$ ( 450 s.c.c.m) flow. After $\mathrm{H}_{2}$ annealing, the system temperature cooled to $850^{\circ} \mathrm{C}$ and pumped/purged with ultra-high pure $\mathrm{N}_{2}$ at least six times to remove $\mathrm{H}_{2}$ gas. Subsequently the chamber is filled in Ar gas (500 s.c.c.m.) to 200 torr. The chamber was then heated up to $1,725^{\circ} \mathrm{C}$ at $100^{\circ} \mathrm{C} \mathrm{min}^{-1}$ and dwelled at this temperature for $20 \mathrm{~min}$ to grow three layers of graphene within the terraces of substrates via sublimation of silicon on the silicon side of $6 \mathrm{H}-\mathrm{SiC}(0001)$. The system cooled down naturally to room temperature after the growth.

$\mathbf{M o S}_{\mathbf{2}}$-WSe $\mathbf{2}$-EG synthesis. WSe ${ }_{2}$ can be grown on EG either via vapour phase reaction of $\mathrm{WO}_{3}$ and Se powders or via MOCVD ${ }^{24,25}$. The vapour phase reaction utilizes the vapourization of $\mathrm{WO}_{3}$ powders in a ceramic boat placed at the centre of $1^{\prime \prime}$ horizontal hot wall tube reactor with a flow of $\mathrm{H}_{2}$ (10 s.c.c.m.)/Ar ( 90 s.c.c.m.). The EG substrates for $\mathrm{WSe}_{2}$ growth were placed at the downstream side of the tube and heated to $925^{\circ} \mathrm{C}$ at $25^{\circ} \mathrm{C} \mathrm{min}-1$. Samples were held at $925^{\circ} \mathrm{C}$ for $15 \mathrm{~min}$ and then cooled naturally to room temperature. The total pressure throughout the reaction is held at 6 torr. Utilizing MOCVD, $\mathrm{WSe}_{2}$ was synthesized in a vertical cold wall reactor using $\mathrm{W}(\mathrm{CO})_{6}$ and $\left(\mathrm{CH}_{4}\right)_{2} \mathrm{Se}$ precursors. The metallic organic precursors were transported into the reactor by carrier gas of $100 \% \mathrm{H}_{2}$ via a bubbler manifold that allows for controlling each precursor concentration independently. The $\left(\mathrm{CH}_{4}\right)_{2} \mathrm{Se}$ and $\mathrm{W}(\mathrm{CO})_{6}$ were held at temperatures of $22{ }^{\circ} \mathrm{C}$ and $25^{\circ} \mathrm{C}$, respectively, and a pressure of 760 torr. The Se to $\mathrm{W}$ ratio was fixed at 20,000 . The MOCVD growth of $\mathrm{WSe}_{2}$ took place at 800 to $850^{\circ} \mathrm{C}$ with a total pressure of 700 torr. The growth time varied between 15 and $30 \mathrm{~min}$. After the completion of $\mathrm{WSe}_{2}-\mathrm{EG}$ synthesis, the ex situ $\mathrm{MoS}_{2}$ growth via the vapour phase reaction using $\mathrm{MoO}_{3}$ and $\mathrm{S}$ powders was carried out in a horizontal hot wall tube reactor at 700 torr. During the $\mathrm{MoS}_{2}$ growth, $\mathrm{MoO}_{3}$ powder in a ceramic boat placed at the centre of heating zone were heated at $750^{\circ} \mathrm{C}$ for $10 \mathrm{~min}$. After the $\mathrm{MoS}_{2}$ growth, the reactor cooled down to room temperature naturally.

$\mathbf{W S e}_{\mathbf{2}}-\mathbf{M o S e}_{\mathbf{2}}$-EG synthesis. In this case, the processes are similar, but steps reversed. The $\mathrm{MoS}_{2}$ is grown first, followed by an ex situ $\mathrm{WSe}_{2}$ growth via the vapour phase reaction of $\mathrm{WO}_{3}$ and Se. A Se-S ion exchange occurs in the $\mathrm{MoS}_{2}$ converting the $\mathrm{MoS}_{2}$ into $\mathrm{MoSe}_{2}{ }^{27}$. Subsequently, the WSe 2 layers grow on $\mathrm{MoSe}_{2}-\mathrm{EG}$ as the hotzone is held at $950^{\circ} \mathrm{C}$ for $45 \mathrm{~min}$, resulting in $\mathrm{WSe}_{2}-\mathrm{MoSe}_{2}-\mathrm{EG}$ heterostructures.

Sample characterization. The details of characterizations performed on the heterostructures can be found in the Supplementary Note 1.

\section{References}

1. Esaki, L. New phenomenon in narrow germanium p-n Junctions. Phys. Rev. 109, 603-604 (1958).

2. Esaki, L. \& Tsu, R. Superlattice and negative differential conductivity in semiconductors. IBM J. Res. Dev. 14, 61-65 (1970).

3. Mitin, V. V., Kochelap, V. \& Stroscio, M. A. Quantum Heterostructures: Microelectronics and Optoelectronics (Cambridge Univ. Press, 1999).

4. Chan, H. L., Mohan, S., Mazumder, P. \& Haddad, G. I. Compact multiple-valued multiplexers using negative differential resistance devices. IEEE J. Solid-State Circuits 31, 1151-1156 (1996).

5. Bayram, C., Vashaei, Z. \& Razeghi, M. AlN/GaN double-barrier resonant tunnelling diodes grown by metal-organic chemical vapor deposition. Appl. Phys. Lett. 96, 042103 (2010).

6. Novoselov, K. S. et al. Electric field effect in atomically thin carbon films. Science 306, 666-669 (2004).

7. Novoselov, K. S. et al. Two-dimensional atomic crystals. Proc. Natl Acad. Sci. USA 102, 10451-10453 (2005).
8. Geim, A. K. \& Grigorieva, I. V. Van der Waals heterostructures. Nature 499, 419-425 (2013).

9. Zhan, Y., Liu, Z., Najmaei, S., Ajayan, P. M. \& Lou, J. Large-area vapor-phase growth and characterization of $\mathrm{MoS}_{2}$ atomic layers on a $\mathrm{SiO}_{2}$ substrate. Small 8, 966-971 (2012).

10. Lee, Y.-H. et al. Synthesis of large-area $\mathrm{MoS}_{2}$ atomic layers with chemical vapor deposition. Adv. Mater. 24, 2320-2325 (2012).

11. Gutiérrez, H. R. et al. Extraordinary room-temperature photoluminescence in triangular $\mathrm{WS}_{2}$ monolayers. Nano Lett. 13, 3447-3454 (2013).

12. Liu, K.-K. et al. Growth of large-area and highly crystalline $\mathrm{MoS}_{2}$ thin layers on insulating substrates. Nano Lett. 12, 1538-1544 (2012).

13. Zhang, Y. et al. Direct observation of the transition from indirect to direct bandgap in atomically thin epitaxial $\mathrm{MoSe}_{2}$. Nat. Nanotechnol. 9, 111-115 (2014).

14. Ugeda, M. M. et al. Giant bandgap renormalization and excitonic effects in a monolayer transition metal dichalcogenide semiconductor. Nat. Mater. 13, 1091-1095 (2014).

15. Terrones, H., López-Urías, F. \& Terrones, M. Novel hetero-layered materials with tunable direct band gaps by sandwiching different metal disulfides and diselenides. Sci. Rep. 3, 1549 (2013).

16. Gao, G. et al. Artificially stacked atomic layers: toward new van der Waals solids. Nano Lett. 12, 3518-3525 (2012).

17. Roy, T. et al. Field-effect transistors built from all two-dimensional material components. ACS Nano 8, 6259-6264 (2014).

18. Britnell, L. et al. Field-effect tunnelling transistor based on vertical graphene heterostructures. Science 335, 947-950 (2012).

19. Haigh, S. J. et al. Cross-sectional imaging of individual layers and buried interfaces of graphene-based heterostructures and superlattices. Nat. Mater. 11, 764-767 (2012).

20. Fang, H. et al. Strong interlayer coupling in van der Waals heterostructures built from single-layer chalcogenides. Proc. Natl Acad. Sci. USA 111, 6198-6202 (2014).

21. Rivera, P. et al. Observation of long-lived interlayer excitons in monolayer $\mathrm{MoSe}_{2}-\mathrm{WSe}_{2}$ heterostructures. Nat. Commun. 6, 6242 (2015).

22. Chiu, M.-H. et al. Spectroscopic signatures for interlayer coupling in $\mathrm{MoS}_{2}-\mathrm{WSe}_{2}$ van der Waals stacking. ACS Nano 8, 9649-9656 (2014).

23. Robinson, J. A. et al. Epitaxial graphene transistors: enhancing performance via hydrogen intercalation. Nano Lett. 11, 3875-3880 (2011).

24. Huang, J.-K. et al. Large-area synthesis of highly crystalline $\mathrm{WSe}_{2}$ monolayers and device applications. ACS Nano 8, 923-930 (2014).

25. Eichfeld, S. M. et al. Highly scalable, atomically thin $\mathrm{WSe}_{2}$ grown via metal-organic chemical vapor deposition. ACS Nano 9, 2080-2087 (2015).

26. Lin, Y.-C. et al. Direct synthesis of van der Waals solids. ACS Nano 8, 3715-3723 (2014).

27. $\mathrm{Su}, \mathrm{S}$.-H. et al. Band gap-tunable molybdenum sulfide selenide monolayer alloy. Small 10, 2589-2594 (2014).

28. Lee, G.-H. et al. Electron tunnelling through atomically flat and ultrathin hexagonal boron nitride. Appl. Phys. Lett. 99, 243114 (2011).

29. Gong, Y. et al. Vertical and in-plane heterostructures from $\mathrm{WS}_{2} / \mathrm{MoS}_{2}$ monolayers. Nat. Mater. 13, 1135-1142 (2014).

30. Wang, Q. H., Kalantar-Zadeh, K., Kis, A., Coleman, J. N. \& Strano, M. S. Electronics and optoelectronics of two-dimensional transition metal dichalcogenides. Nat. Nanotechnol. 7, 699-712 (2012).

31. Chiu, M.-H. et al.Determination of band alignment in transition metal dichalcogenides heterojunctions (2014; Preprint at $<$ http://arxiv.org/abs/ $1406.5137>$ ).

32. Yang, W. et al. Epitaxial growth of single-domain graphene on hexagonal boron nitride. Nat. Mater. 12, 792-797 (2013).

33. Parkinson, B. A., Ohuchi, F. S., Ueno, K. \& Koma, A. Periodic lattice distortions as a result of lattice mismatch in epitaxial films of two-dimensional materials. Appl. Phys. Lett. 58, 472-474 (1991).

34. Klein, A., Tiefenbacher, S., Eyert, V., Pettenkofer, C. \& Jaegermann, W. Electronic band structure of single-crystal and single-layer $\mathrm{WS}_{2}$ : Influence of interlayer van der Waals interactions. Phys. Rev. B 64, 205416 (2001).

35. Zhang, C., Johnson, A., Hsu, C.-L., Li, L.-J. \& Shih, C.-K. Direct imaging of band profile in single layer $\mathrm{MoS}_{2}$ on graphite: quasiparticle energy gap, metallic edge states, and edge band bending. Nano Lett. 14, 2443-2447 (2014).

36. Rawlett, A. M. et al. Electrical measurements of a dithiolated electronic molecule via conducting atomic force microscopy. Appl. Phys. Lett. 81, 3043-3045 (2002).

37. Georgiou, T. et al. Vertical field-effect transistor based on graphene-WS heterostructures for flexible and transparent electronics. Nat. Nanotechnol. 8, 100-103 (2013).

38. Roy, T. et al. Dual-gated $\mathrm{MoS}_{2} / \mathrm{WSe}_{2}$ van der waals tunnel diodes and transistors. ACS Nano 9, 2071-2079 (2015).

39. Lee, C.-H. et al. Atomically thin p-n junctions with van der Waals heterointerfaces. Nat. Nanotechnol. 9, 676-681 (2014). 
40. Smet, J. H., Broekaert, T. P. E. \& Fonstad, C. G. Peak-to-valley current ratios as high as 50:1 at room temperature in pseudomorphic $\operatorname{In}_{0.53} \mathrm{Ga}_{0.47} \mathrm{As} / \mathrm{AlAs} / \mathrm{InAs}$ resonant tunnelling diodes. J. Appl. Phys. 71, 2475 (1992).

41. Day, D. J., Yang, R. Q., Lu, J. \& Xu, J. M. Experimental demonstration of resonant interband tunnel diode with room temperature peak-to-valley current ratio over 100. J. Appl. Phys. 73, 1542 (1993).

42. Su, Y.-K. et al. Well width dependence for novel AlInAsSb/InGaAs double-barrier resonant tunnelling diode. Solid-State Electron. 46, 1109-1111 (2002).

43. Tsai, H. H., Su, Y. K., Lin, H. H., Wang, R. L. \& Lee, T. L. P-N double quantum well resonant interband tunnelling diode with peak-to-valley current ratio of 144 at room temperature. IEEE Electron Dev. Lett. 15, 357-359 (1994).

44. Evers, N. et al. Thin film pseudomorphic AlAs/ $\mathrm{In}_{0.53} \mathrm{Ga}_{0.47} \mathrm{As} / \mathrm{InAs}$ resonant tunnelling diodes integrated onto Si substrates. IEEE Electron Dev. Lett. 17, 443-445 (1996).

45. Rommel, S. L. et al. Epitaxially grown Si resonant interband tunnel diodes exhibiting high current densities. IEEE Electron Dev. Lett. 20, 329-331 (1999).

46. See, P. et al. High performance $\mathrm{Si} / \mathrm{Si}_{1-\mathrm{x}} / \mathrm{Ge}_{\mathrm{x}}$ resonant tunnelling diodes. IEEE Electron Dev. Lett. 22, 182-184 (2001).

47. Jin, N. et al. Diffusion barrier cladding in $\mathrm{Si} / \mathrm{SiGe}$ resonant interband tunnelling diodes and their patterned growth on PMOS source/drain regions. IEEE Trans. Electron Dev. 50, 1876-1884 (2003).

48. Britnell, L. et al. Resonant tunnelling and negative differential conductance in graphene transistors. Nat. Commun. 4, 1794 (2013).

49. Mishchenko, A. et al. Twist-controlled resonant tunnelling in graphene/boron nitride/graphene heterostructures. Nat. Nanotechnol. 9, 808-813 (2014).

\section{Acknowledgements}

Support is acknowledged by the Center for Low Energy Systems Technology (LEAST), one of six centers supported by the STARnet phase of the Focus Center Research Program (FCRP), a Semiconductor Research Corporation program sponsored by MARCO and DARPA. Work at UT-Dallas was also supported by the Southwest
Academy on Nanoelectronics (SWAN) a SRC center sponsored by the Nanoelectronics Research Initiative and NIST. The WiteC Raman system and Bruker Dimension AFM, and nanofabrication facilities were supported by the National Nanotechnology Infrastructure Network at the Penn State.

\section{Author contributions}

J.A.R. and Y.-C.L. conceived the idea, and J.A.R., S.D., R.M.W., M.J.K. and L.-J.L. directed the research. Y.-C.L., M.-Y.L. and S.M.E. synthesized the heterostructures. Y.-C.L. carried out AFM, Raman, photoluminescence and CAFM measurements. R.A. carried out STM/STS; H.Z. and X.P. carried out XPS; N.L. carried out TEM; and R.K.G. carried out the modelling. Y.-C.L. and J.A.R. wrote the paper with significant inputs from all authors. All authors have read and approved the manuscript. All authors participated in the analysis of the data and discussed the results.

\section{Additional information}

Supplementary Information accompanies this paper at http://www.nature.com/ naturecommunications

Competing financial interests: The authors declare no competing financial interests.

Reprints and permission information is available online at http://npg.nature.com/ reprintsandpermissions/

How to cite this article: Lin, Y-C. et al. Atomically thin resonant tunnel diodes built from synthetic van der Waals heterostructures. Nat. Commun. 6:7311 doi: $10.1038 /$ ncomms8311 (2015).

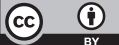

This work is licensed under a Creative Commons Attribution 4.0 International License. The images or other third party material in this article are included in the article's Creative Commons license, unless indicated otherwise in the credit line; if the material is not included under the Creative Commons license, users will need to obtain permission from the license holder to reproduce the material. To view a copy of this license, visit http://creativecommons.org/licenses/by/4.0/ 